\title{
Cooperación al desarrollo en el Bajo Lempa (El Salvador): percepción del estado de salud y condición nutricional
}

\section{Development Cooperation in Bajo Lempa (El Salvador): Perception of the Health and Nutritional Condition}

Roberto Pedrero-Tomé ${ }^{1,2}$, María Sánchez Álvarez ${ }^{1,2}$,
Noemí López-Ejeda ${ }^{1,2}$, Cristina Herrero-Jáureguil,
Belén Acosta Gallo y María Dolores Marrodán

\section{Resumen}

En la región salvadoreña del Bajo Lempa se han elaborado los proyectos de cooperación al desarrollo sostenible de la Universidad Complutense de Madrid (ref. 19/17, 18/11), en el que se analiza el nivel de calidad de vida de la zona mediante la condición nutricional de la población escolar y el nivel de seguridad alimentaria. Se evaluó la percepción sobre el estado de salud de 27 productores en función del tipo de agua de consumo; el estado nutricional de 334 escolares (5-16 años) y la percepción de seguridad alimentaria en 143 hogares. Los resultados obtenidos informan de la existencia de 59,7\% de hogares con algún tipo de inseguridad alimentaria, siendo la tipología grave la que mayor porcentaje aporta $(25,9 \%)$. Se detecta una asociación positiva entre el nivel educativo materno y el nivel de seguridad alimentaria. Se reporta $23,8 \%$ de sobrecarga ponderal y $9 \%$ de desnutrición crónica.

Palabras clave: desnutrición crónica, EPSA, El Salvador, seguridad alimentaria, sobrecarga ponderal.

Grupo de Investigación EPINUT (ref. 920325). Universidad Complutense de Madrid, España.

2 Departamento de Biodiversidad, Ecología y Evolución. Universidad Complutense de Madrid, España.

3 Instituto Universitario de Ciencias Ambientales (IUCA). Universidad Complutense de Madrid, España. 


\section{Abstract}

In the Salvadoran region of Bajo Lempa, sustainable development cooperation projects have been carried out by the Universidad Complutense de Madrid (ref. 19/17, $18 / 11$ ) to analyze the quality of life in the area through the nutritional condition of the school population and the level of food security. We assessed the perception of the health condition of 27 producers according to the type of drinking water, the nutritional status of 334 schoolchildren (5-16 years), and the perception of food security in 143 households. The results show $59.7 \%$ of households with food insecurity, the severe type contributing the highest percentage $(25.9 \%)$. A positive association is detected between the maternal educational level and the level of food security. Besides, $23.8 \%$ overweight and $9 \%$ chronic malnutrition are reported.

Keywords: Chronic malnutrition; EPSA; El Salvador; food safety; overweight.

\section{Introducción}

La región salvadoreña del Bajo Lempa es reconocida como un área crítica en términos de amenaza, riesgo y vulnerabilidad, debido a las inundaciones de los terrenos adyacentes al curso del agua en los episodios de lluvias torrenciales, a la presencia de aguas no tratadas y al empleo de cantidades ingentes de agrotóxicos en determinados cultivos (Johnson y Sánchez, 2013).

Los procesos de desarrollo rural en este territorio se encuentran fuertemente condicionados por la existencia de dos modelos de producción contrapuestos, que compiten por los mismos recursos naturales. Por una parte, un patrón de producción familiar caracterizado por cultivos diversificados que tienen como finalidad el autoabastecimiento y, en determinadas ocasiones, el comercio con intermediarios. Por otra, grandes extensiones de cultivo de caña de azúcar mayoritariamente pertenecientes al sector privado. Otro factor que puede condicionar el desarrollo rural es la fuente de origen de agua. Aún no se ha conseguido garantizar un suministro de agua potable y segura en determinadas regiones rurales de América Latina y el Caribe (Leigh, 2017). Por ello, es muy probable que algunas comunidades asentadas en la región salvadoreña del Bajo Lempa aún se abastezcan de aguas superficiales, de pozos excavados, de entregas de camiones cisterna o de agua embotellada. 
La alimentación es otro factor que puede condicionar el estado de salud. Tal y como apunta la Organización de las Naciones Unidas para la Alimentación y la Agricultura (FAO, 2019), se está produciendo un incremento en las formas de malnutrición por exceso en los países de bajos y medianos ingresos, como El Salvador, como consecuencia de la baja disponibilidad de alimentos saludables y el escaso acceso a una alimentación saludable y equilibrada.

Con la finalidad de mejorar la productividad agrícola y la calidad de vida en las comunidades del Bajo Lempa, durante el 2018 y 2019 se desarrollaron dos proyectos de Cooperación al Desarrollo Sostenible, entre la Universidad Complutense de Madrid, la Universidad Paracentral de El Salvador y otras organizaciones e instituciones como la Alcaldía de Tecoluca, la Fundación del Valle y la Asociación-Fundación para la Cooperación y el Desarrollo Comunal de E1 Salvador (Cordes) (ref. 17/19, 18/11).

La presente investigación tiene como objetivo establecer una línea de base que sirva a futuro para comprobar si las acciones planteadas en los proyectos de cooperación definidos contribuyen a mejorar la calidad de vida, empleando como indicadores objetivos la percepción del estado de salud, el tipo de agua de consumo, la seguridad alimentaria y la condición nutricional de la población escolar.

Se plantea un estudio descriptivo y de asociación entre variables indicativas de la condición nutricional, abastecimiento de agua y percepción de la salud y la seguridad alimentaria.

\section{Material y métodos}

\section{Estado de salud de los productores locales en función del origen del agua de consumo}

Se entrevistó a 27 productores locales (17 varones y 10 mujeres) con edades comprendidas entre los 28 y 89 años (Media: 53,71; DE: 14,49), cumplimentando el cuestionario de percepción de la salud y calidad de vida EQ5D (Johnson et al., 1998). Se tomaron datos de peso (kg) y estatura $(\mathrm{cm})$, calculando el índice de masa corporal (IMC) y clasificando su estado nutricional mediante el criterio de la Organización Mundial de la Salud (OMS, 2020). Asimismo, se recabó información sobre la fuente de agua para el consumo. Cabe destacar que todos los productores mantienen sus cultivos de manera orgánica, gracias a diversos proyectos promovidos por la Cordes. 


\section{Caracterización socioeconómica y evaluación de la percepción de la seguridad alimentaria}

Se realizaron 143 entrevistas semiestructuradas a las madres y padres de los escolares en las que se preguntó sobre el número de menores que conforman la unidad familiar, el nivel máximo de estudios y la situación laboral de las madres y padres, así como el nivel de seguridad alimentaria del hogar mediante la Encuesta de Percepción de la Seguridad Alimentaria (EPSA) (Wehler et al., 1992). Las respuestas a la misma permitieron clasificar los hogares como seguros (0 puntos), con inseguridad leve (1-7 puntos), moderada (8-14 puntos) o grave ( $\geq 15$ puntos).

\section{Condición nutricional en la población infantil}

Con el consentimiento informado de progenitores o tutores legales y de acuerdo con la normativa de la Asociación Médica Mundial (AMM, 2013), se evaluó la condición nutricional de 334 escolares (176 niñas y 158 niños) entre 5 y 16 años. En las escuelas se tomaron datos antropométricos de peso $(\mathrm{kg})$ y estatura $(\mathrm{cm})$, calculando el IMC para clasificar a los escolares en insuficiencia ponderal (IP), normopeso (NP), sobrepeso (SP) y obesidad (OB) tomando como criterio el patrón de la OMS (2007). Utilizando las mismas curvas de crecimiento como referencia se comprobó si el crecimiento longitudinal era normal o si los niños padecían desnutrición crónica.

\section{Procedimiento estadístico}

Se aplicaron pruebas de Chi-cuadrado para el contraste de proporciones y regresión logística para asociar factores sociodemográficos al estado de seguridad alimentaria. El tratamiento de los datos se efectuó mediante el software IBM SPSS 23.0.

\section{Resultados}

\section{Recursos hídricos y estado de salud de los productores}

El 100\% de los encuestados refirió abastecerse de agua tratada de diferentes fuentes y orígenes: ocho productores $(29,63 \%)$ obtienen agua canalizada procedente del pozo de la comunidad de Guajoyo y el 70,37\% restante se abastece de agua potable de la región de San Carlos Lempa, San Nicolás o consume agua embotellada para beber.

Los productores que consumen agua potable o embotellada tienden a presentar o percibir mejor estado de salud mental y menor dificultad para realizar las tareas cotidianas que los sujetos que 
consumen agua de pozo tratada (tabla 1). La condición nutricional no guardó ninguna relación con el tipo de abastecimiento hídrico, siendo la proporción de IP del 4,2\%, 37,5\% de NP, 35,4\% de SP y $22,9 \%$ de OB $\left(\chi^{2}: 2,737 ; p=0,434\right)$.

\section{Determinantes de la seguridad alimentaria en los hogares}

La figura 1 muestra la estrecha asociación entre el nivel educativo y el tipo de trabajo maternos con la seguridad alimentaria. Los hogares en los que la mujer posee estudios secundarios o superiores son significativamente más se$\operatorname{guros}\left(\chi^{2}: 20,932 ; p<0,001\right)$ frente a aquellos en los que las mujeres no alcanzan o se quedan en la educación primaria. De mismo modo, cuando las mujeres tienen un trabajo remunerado externo, el hogar tiende a ser más seguro $\left(\chi^{2}: 3,009\right.$; $p=0,083)$.

Tabla 1. Estado de salud de los productores locales en función de la fuente de consumo de agua

\begin{tabular}{|c|c|c|c|c|}
\hline & Agua potable & $\begin{array}{c}\text { Agua tratada } \\
\text { y canalizada }\end{array}$ & $\chi^{\mathbf{2}}$ & $\boldsymbol{p}$ \\
\hline Experiencia en enfermedades en sí mismo & $15,8 \%$ & $37,5 \%$ & 1,535 & 0,215 \\
\hline Sentimiento de ansiedad o depresión & $15,8 \%$ & $25,0 \%$ & 0,317 & 0,574 \\
\hline Dificultad para realizar las actividades diarias & $15,8 \%$ & $50,0 \%$ & 3,431 & 0,064 \\
\hline Toma algún medicamento de manera crónica & $36,8 \%$ & $50,0 \%$ & 0,404 & 0,525 \\
\hline
\end{tabular}

Fuente: elaboración propia.

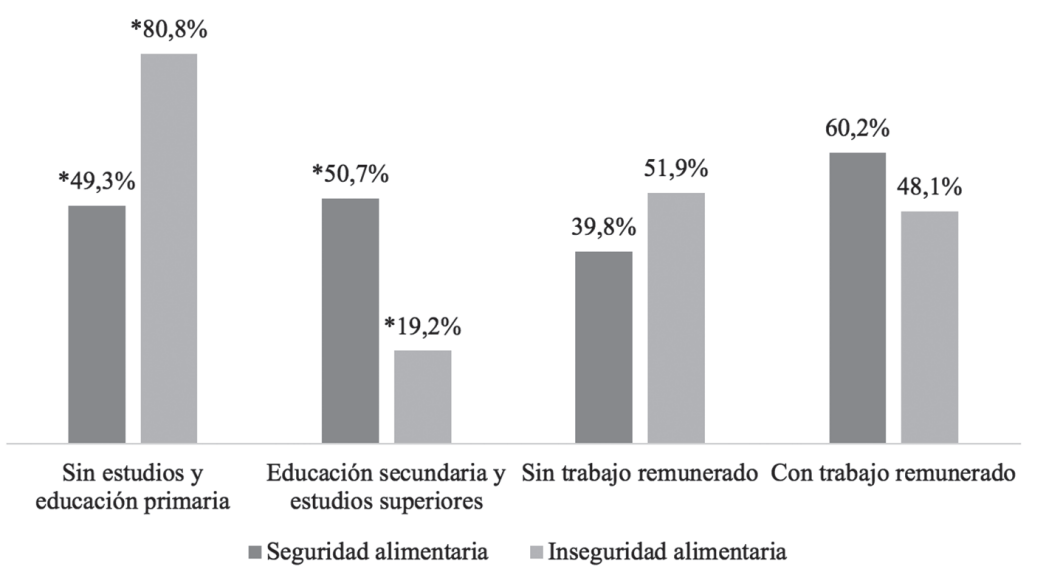

Figura 1. Nivel de seguridad alimentaria en el hogar en función del nivel de estudios y el tipo de trabajo realizado por las madres $\left({ }^{*} p<0,001\right)$

Fuente: elaboración propia. 
En la tabla 2 se recoge el nivel de seguridad alimentaria en función de la cantidad de menores de edad que conviven en el mismo hogar. Se observa que el grado "seguridad alimentaria" disminuye entre el primero y segundo hijo, para aumentar significativamente a partir del quinto $\left(\chi^{2}: 24,966 ; p<0,001\right)$.

Tabla 2. Nivel de seguridad alimentaria en función del número de menores que conviven en el mismo hogar

\begin{tabular}{|c|c|c|c|c|}
\hline $\begin{array}{c}\text { Tipo de hogar } \\
\text { según EPSA }\end{array}$ & $\mathbf{N = 1}$ & $\mathbf{N = \mathbf { 2 }}$ & $\mathbf{N = 3 - 4}$ & $\mathbf{N}=+\mathbf{5}$ \\
\hline $\begin{array}{c}\text { Con seguridad } \\
\text { alimentaria }\end{array}$ & $43,3 \%$ & $20,0 \%$ & $39,7 \%$ & $65,2 \%$ \\
\hline $\begin{array}{c}\text { Inseguridad } \\
\text { alimentaria }\end{array}$ & $56,7 \%$ & $80,0 \%$ & $60,3 \%$ & $34,8 \%$ \\
\hline $\mathrm{p}$ & \multicolumn{3}{|c|}{$<0,001$} \\
\hline
\end{tabular}

Fuente: elaboración propia.

\section{Seguridad alimentaria y condición nutricional de la población infantil}

En la población escolar se detectó $15,4 \%$ de IP, $60,8 \%$ de NP y $22,5 \%$ de sobrecarga ponderal $(13,8 \%$ de SP y $10 \%$ de $\mathrm{OB}$ ), mientras que la prevalencia de la baja talla para la edad o desnutrición crónica fue del 9\%.

El $59,7 \%$ de los hogares fueron catalogados como inseguros, de los cuales al $25,9 \%$ le correspondió el tipo grave.
El modelo de regresión mostró una relación negativa entre la edad $(-0,142)$ y el número de menores que conforman la unidad convivencial $(-0,342)$ respecto al nivel de seguridad alimentaria en el hogar $\left(\mathrm{R}^{2}\right.$ de Nagelkerke: 0,116; $p<$ $0,005)$. Así, el riesgo de padecer inseguridad alimentaria disminuye $14,2 \%$ (OR $=0,858 ; \mathrm{IC}=95 \%$ : 0,776-0,949) cuando se incrementa en un año la edad de los menores y $13,8 \%(\mathrm{OR}=0,862$; IC $=95 \%: 0,741-1,003)$ cuando se incrementa en uno el número de menores que conviven en el mismo hogar.

\section{Discusión}

Pese a que no existen diferencias significativas en el estado de salud de los productores en función del origen de los recursos hídricos, sí se observa una tendencia hacia la percepción de un mejor estado de salud de quienes consumen agua potable o embotellada.

Se reporta una elevada proporción de hogares con inseguridad alimentaria. El comportamiento y las decisiones que repercuten sobre la nutrición dependen de numerosos factores, entre los que podemos destacar: el contexto económico y social, la oferta alimentaria, el cambio climático, la disponibilidad ecológica de los alimentos y el nivel educativo (Parás 
y Pérez-Escamilla, 2004). De hecho, en el presente trabajo se detecta una mayor proporción de hogares nutricionalmente seguros cuando la mujer ha podido cursar estudios superiores y cuando obtiene una remuneración por su trabajo, ya que todos los hogares analizados cuentan como mínimo con el salario del padre de familia. Es importante señalar que el hecho de que las mujeres accedan a una educación de mejor calidad, repercutirá de manera directa sobre el control de la seguridad alimentaria en el hogar; puesto que, por norma general, son las responsables del cuidado de la alimentación y la transmisión de los conocimientos culinarios a sus descendientes (Álvarez-Castaño et al., 2012). Además, cuando un hogar cuenta con más hijos y estos son de mayor edad, se reduce el riesgo de inseguridad alimentaria. Posiblemente, porque los hijos mayores contribuyen al cuidado de los pequeños y al mantenimiento de la familia, como es frecuente en poblaciones rurales (Bonilla-Aparicio et al., 2013).

La condición nutricional observada en la población escolar y adulta coincide con la reportada por la Política Nacional de Seguridad Alimentaria (2018-2028), documento que ya alertaba del incremento de la sobrecarga ponderal en todas las franjas etarias y de la existencia de desnutrición crónica o retardo en el crecimiento en la población infantil y escolar (Conasan, 2018). Todas estas cifras permiten visibilizar el fenómeno de la doble carga de malnutrición tan característico de sociedades en transición nutricional, en las que se frecuenta el consumo de alimentos baratos, de escaso valor nutricional y elevado contenido calórico (Fernández et al., 2017). Otro factor que puede condicionar el patrón de consumo alimentario $y$, por tanto, la condición nutricional, es el proceso de aculturación a distancia en el que se encuentran sumergidos la mayoría de los países de América Latina y el Caribe, como El Salvador. Por este motivo, los individuos pueden modificar sus deseos y preferencias con la finalidad de asemejarse a las personas que viven en países con economías más desarrolladas (Popkin, 2015).

Cabe destacar que el presente estudio se definió como un proyecto piloto, por lo que ha podido encontrarse limitado en algunos sentidos como, por ejemplo, el escaso tamaño muestral de los productores y el empleo de una escala subjetiva como única medida de la percepción del estado de salud. 


\section{Conclusiones}

El consumo de agua potable o embotellada se asocia a una mejor percepción del estado de salud. Más de la mitad de los hogares analizados presentan inseguridad alimentaria, siendo una cuarta parte de la tipología más grave. Se reporta un menor riesgo de inseguridad cuando se incrementa la edad y el número de menores que viven en el hogar. Se observa una asociación positiva en el nivel educativo materno y el nivel de seguridad alimentaria en el hogar. La presencia de exceso de peso y desnutrición crónica o retraso en el crecimiento, permiten visibilizar la doble carga de malnutrición que soportan las comunidades salvadoreñas asentadas en la región del Bajo Lempa.

\section{Agradecimientos}

La presente investigación se enmarca en las Ayudas para Proyectos de Cooperación para el Desarrollo Sostenible de la Universidad Complutense de Madrid. A partir de esta convocatoria, se facilitó la cooperación interuniversitaria entre el Departamento de Biodiversidad, Ecología y Evolución de la Facultad de Biología de la Universidad Complutense de Madrid, el Grupo de Investigación EPINUT-UCM y la Universidad Paracentral de El Salvador. Asimismo, también se agradece la participación, compromiso e implicación de otras organizaciones e instituciones, como la Alcaldía de Tecoluca, la Fundación del Valle y la Asociación-Fundación para la Cooperación y el Desarrollo Comunal de El Salvador (Cordes), ya que sin su colaboración este proyecto no habría sido posible.

\section{Referencias}

Álvarez-Castaño, L. S., Goez-Rueda, J. D. y Carreño-Aguirre, C. (2012). Factores sociales y económicos asociados a la obesidad: los efectos de la inequidad y de la pobreza. Revista Gerencia y Políticas de Salud, 11(23), 98-110.

AMM. Asociación Médica Mundial. (2013). 64a Asamblea General. Declaración de Helsinki. Principios éticos para las investigaciones médicas en seres humanos. https://www.wma.net/es/policies-post/ declaracion-de-helsinki-de-la-amm-principios-eticos-para-las-investigaciones-medicas-en-seres-humanos/

Bonilla-Aparicio, M. E., Salcido-Ramos, B. A., Paredes-Sánchez, J. A., Aguirre-Álvarez, L., Méndez-Cadena, M. E. y Hernández-Rodríguez, M. L. (2013). La diversidad hortícola para la seguridad alimentaria en municipios marginados del estado de Puebla. Ra Ximhai, 9(2), 151-163.

Conasan. Consejo Nacional de Seguridad Alimentaria y Nutricional. (2018). Politica Nacional de Seguridad Alimentaria y Nutricional (2018-2028). El Salvador: Conasan. 
Cordes. Asociación-Fundación para la Cooperación y el Desarrollo Comunal de El Salvador. (2017). Diagnóstico participativo. Área de desarrollo territorial, Jaltepeque Bahía. El Salvador: Cordes.

FAO. Organización de las Naciones Unidas para la Alimentación y la Agricultura. (2019). El estado de la seguridad alimentaria y la nutrición en el mundo. Roma: FAO. http:// www.fao.org/3/ca5162es/ca5162es.pdf

Fernández, A., Martínez, R., Carrasco, I. y Palma, A. (2017). Impacto social y económico de la malnutrición. Modelo de análisis y estudio piloto en Chile, el Ecuador y México. Santiago, Chile: Programa Mundial de Alimentos, Naciones Unidas. https:// repositorio.cepal.org/bitstream/handle/11362/42535/1/S1700443_es.pdf

Johnson, J. A., Coons, S. J., Ergo, A., \& Szava-Kovats, G. (1998). Valuation of EuroQol (EQ-5D) health states in an adult US sample. Pharmacoeconomics, 13(4), 421433. doi: 10.2165/00019053-19981304000005 .

Johnson, R. J., \& Sánchez, L. G. (2013). Chronic kidney disease: Mesoamerican nephropathy-new clues to the cause. Nature Reviews Nephrology, 9(10), 560-561. doi: 10.1038/nrneph.2013.174.
Leigh, N. D. (2017). Lessons from the field: Humanitarian work in Latin America. En: Ronda L. Grosse (ed.). Mobilizing chemistry expertise to solve humanitarian problems (pp. 99-115). American Chemical Society. doi: 10.1021/bk-2017-1267.ch007

OMS. Organización Mundial de la Salud. (2007). Referencias de crecimiento de 5-19 años. https://www.who.int/growthref/en/

OMS. Organización Mundial de la Salud. (2020). Datos y cifras. 10 datos sobre la obesidad. https://www.who.int/features/ factfiles/obesity/facts/es/

Parás, P. y Pérez-Escamilla. R. (2004). El rostro de la pobreza: la inseguridad alimentaria en el Distrito Federal. Revista Este País, 158(5), 45-50.

Popkin, B. M. (2015). Nutrition transition and the global diabetes epidemic. Current Diabetes Reports, 15(9), 1-8. doi: 10.1007/ s11892-015-0631-4.

Wehler, C. A., Scott, R. I., \& Anderson, J. J. (1992). The community Childhood hunger identification project: A model of domestic hunger-demonstration project in Seattle, Washington. Journal of Nutrition Education, 24(1), 29-35. 